\title{
Flow simulation and aerodynamic noise prediction for a high-speed train wheelset
}

\author{
J. Y. Zhu', Z. W. Hu' and D. J. Thompson ${ }^{2}$ \\ 'Aerodynamics and Flight Mechanics Research Group, Faculty of Engineering and the Environment, \\ University of Southampton, Southampton SO17 1BJ, United Kingdom \\ 2Institute of Sound and Vibration Research, Faculty of Engineering and the Environment, University \\ of Southampton, Southampton SO17 1BJ, United Kingdom
}

Received: Feb. 16, 2014; Revised: Aug. 18, 2014; Accepted: Sept. 1, 2014

\begin{abstract}
Aerodynamic noise becomes significant for high-speed trains and its prediction in an industrial context is hard to achieve. The aerodynamic and aeroacoustic behaviour of the flow past a highspeed train wheelset, one of the main components of a bogie, are investigated at a scale 1:10 using a two-stage hybrid method of computational fluid dynamics and acoustic analogy. The near-field unsteady flow is obtained by solving the Navier-Stokes equations numerically through delayed detached-eddy simulations and the results are fed to predict the far-field noise signals using the Ffowcs Williams-Hawkings acoustic analogy. Far-field sound radiated from the scaled model is also measured in a low noise open-jet anechoic wind tunnel. Good agreement is achieved between numerical and experimental results for the dominant frequency of tonal noise and the shape of the spectra. Results show that turbulent flow past the wheelset is characterized by three-dimensional streamwise and spanwise vortices with various scales and orientations. Vortex shedding and flow separation around the wheelset are the key factors for the aerodynamic noise generation. It is found that the radiated tonal noise corresponds to the dominant frequencies of the oscillating lift and drag forces from the wheelset. The directivity of the noise radiated exhibits a typical dipole pattern. As the inflow velocity increases, the shedding frequency scales with the freestream velocity and the axle diameter to yield a Strouhal number of 0.18 while the noise levels increase noticeably. For the current wheelset case investigated without considering the ground effect, the inclusion of wheelset rotation increases the radiated noise levels slightly with similar directivity.
\end{abstract}

\section{INTRODUCTION}

For high-speed trains, it is generally accepted that the aerodynamic noise becomes

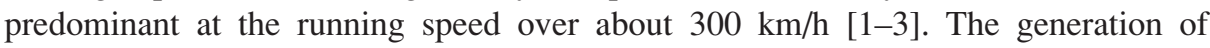
aerodynamic noise from high-speed trains is less well understood and numerical calculations have been restricted to some simple geometries using the traditional

*E-mail addresses: jz1e10@soton.ac.uk (J. Y. Zhu), z.hu@soton.ac.uk (Z.W. Hu), djt@isvr.soton.ac.uk (D. J. Thompson) 
computational methods [3]. The flow-induced noise from a full-scale simplified highspeed train was studied numerically using the lattice-Boltzmann method along with the turbulence wall-function approach, although verification by experimental measurements is required to improve confidence [4]. By comparison, the calculations on some simplified scaled geometries can reveal more details of the flow behaviour and the corresponding aeroacoustic mechanisms in some main components of high-speed trains. Moreover, these numerical simulations can be performed with affordable computer resources and verified by experimental measurements. Results from these model cases can be used to determine the relative importance of various aerodynamic noise sources and to establish efficient noise prediction methods, such as the component-based model developed to predict the aerodynamic noise from high-speed trains [5].

It is still very difficult to predict aerodynamic noise in an industrial context due to large computational resources required for unsteady numerical simulations [6]. This research aims to study the aerodynamic noise generation mechanism from the scale models with increased complexity around high-speed train bogie regions and to see the differences between the various cases. As an initial step, this paper focuses on investigating the flow behaviour and the corresponding aerodynamic noise characteristics from an isolated wheelset of a high-speed train: two wheels attached to an axle. This can also be represented as a general case of circular cylinder with two discs fixed close to the ends, which is of some practical applications. Calculations are performed for a 1:10 scale wheelset and its components. Numerical results of the aerodynamic noise are verified by the experimental measurements from an anechoic open-jet wind tunnel.

\section{NUMERICAL METHOD}

Numerical simulations are carried out using a two-stage strategy of computational fluid dynamics (CFD) and computational aeroacoustics (CAA) methods. Aerodynamically, high-speed trains are operating within the low Mach number flow regime. The incoming flow simulated here is also at low Mach numbers ( 0.09 and 0.2 corresponding to 30 and $70 \mathrm{~m} / \mathrm{s}$ ) and thereby the compressibility effects may be neglected to the hydrodynamic flow field. Moreover, at low Mach number the dominant noise sources are the dipole sources from wall pressure fluctuations, which can be predicted essentially through incompressible flow modelling. Therefore, the unsteady, incompressible Navier-Stokes equations are used to solve the flow field. The open source software OpenFOAM-2.2.1 is employed to solve the governing equations. A second-order accurate scheme is used for the spatial derivatives and the temporal discretisation follows a second-order fully implicit scheme. The delayed detached-eddy simulation (DDES) based on the Spalart-Allmaras turbulence model is employed for the current flow calculations [7].

The near-field unsteady flow computation provides acoustic sources which are fed to Ffowcs Williams-Hawkings (FW-H) acoustic analogy for far-field noise prediction $[8,9]$. The formal solution of the FW-H equation may be written as 


$$
\begin{array}{r}
p^{\prime}(\mathbf{x}, t)=\frac{\partial}{\partial t} \int_{f=0}\left[\frac{Q_{i} n_{i}}{4 \pi|\mathbf{x}-\mathbf{y}|}\right]_{\tau_{e}} d s-\frac{\partial}{\partial x_{i}} \int_{f=0}\left[\frac{L_{i j} n_{j}}{4 \pi|\mathbf{x}-\mathbf{y}|}\right]_{\tau_{e}} d s \\
+\frac{\partial^{2}}{\partial x_{i} x_{j}} \int_{f>0}\left[\frac{T_{i j}}{4 \pi|\mathbf{x}-\mathbf{y}|}\right]_{\tau_{e}} d V
\end{array}
$$

where $f(\mathbf{x}, t)=0$ describes the control surface. [ $]_{\tau_{e}}$ denotes the evaluation at the emission time. The acoustic pressure $p^{\prime}(\mathbf{x}, t)$ represents the pressure fluctuation perceived by an observer at observer time $t$. The source terms under the integral sign are defined as

$$
\begin{gathered}
Q_{i}=\rho\left(u_{i}-v_{i}\right)+\rho_{0} v_{i}, \\
L_{i j}=\left(p-p_{0}\right) \delta_{i j}+\rho u_{i}\left(u_{j}-v_{j}\right), \\
T_{i j}=p u_{i} u_{j}-\tau_{i j}+\left[\left(p-p_{0}\right)-c_{0}^{2} \rho^{\prime}\right] \delta_{i j},
\end{gathered}
$$

where $T_{i j}$ is the Lighthill stress tensor; $Q_{i}$ and $L_{i j}$ are thickness and loading noise sources respectively $[9,10] . u_{i}$ and $v_{i}$ are the flow and surface velocity components in the $i$ th direction. $\tau_{i j}$ is the viscous stress tensor and $\delta_{i j}$ the Kronecker delta. Owing to a low Mach number flow simulated, the quadrupole noise from the Lighthill stress tensor may be neglected and Farassat's formulation 1A is employed to solve FW-H equation and predict the noise generated $[9,10]$.

\section{SIMULATION SETUP}

The present analysis is based on a 1:10 scale simplified wheelset model, displayed in Fig. 1, where $x$ is the flow direction, $y$ the vertical direction and $z$ the spanwise direction. Although the shape of a railway wheel is rather complex, wheel-mounted braking systems are often implemented on the power bogie of high-speed trains, enabling the wheel to be represented as a flat-sided disc by neglecting the gap between the wheel and braking discs. The flange of the running surface is also neglected here.

In this $1 / 10^{\text {th }}$ model, the axle has a diameter $(d)$ of $17.5 \mathrm{~mm}$ and the wheels have a diameter $(D)$ of $92 \mathrm{~mm}$. The wheelset geometry is symmetrical along the axle mid-span where the flow influence from the wheel is small. Therefore, it is reasonable to make use of symmetry to cut the computational domain with half the axle and a single wheel. The use of symmetry plane was investigated and demonstrated to be acceptable [4]. The domain has dimensions $15 D \times 10 D \times 6 D$ (length, height and width) where $D$ is the wheel diameter; thus, the blockage ratio (defined as the ratio of the projected wheelset area to the domain cross-sectional area) is small (about $0.5 \%$ ); and the outlet boundary is far enough to have negligible influence on the near-wake flow around the wheelset. 


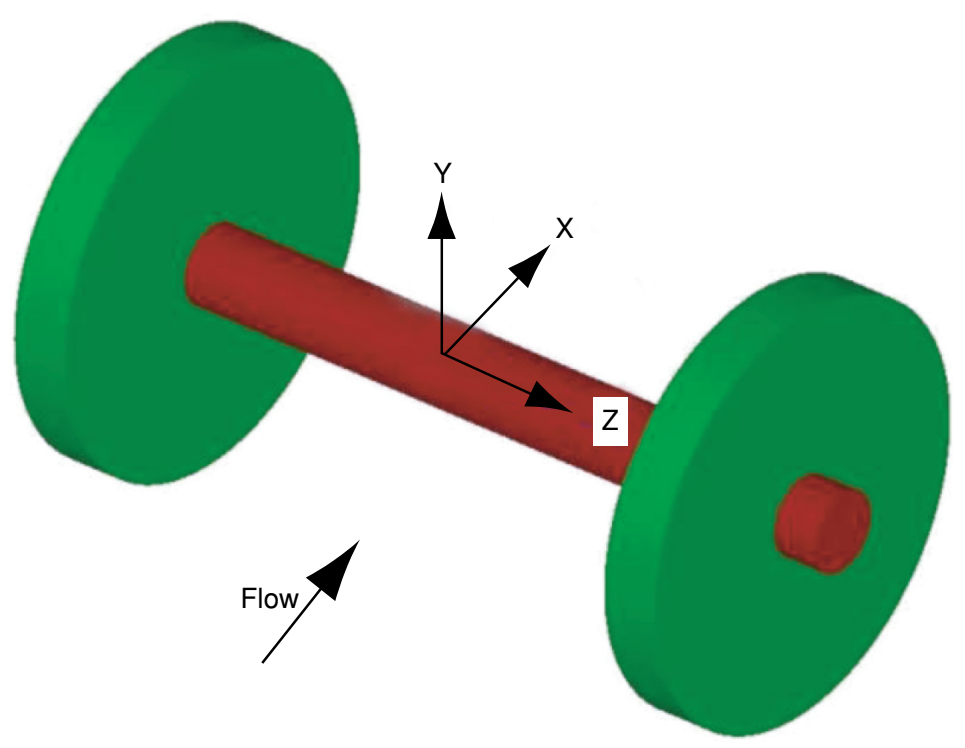

Figure 1: $\quad$ Isolated wheelset model.

The following boundary conditions are applied: the upstream inlet flow is represented as a steady uniform flow $\left(U_{\infty}\right)$ with a low turbulence intensity; the top, bottom, axle mid-plane and side boundaries are specified as symmetry boundaries which are equivalent to zero-shear slip walls and assume no flux of any quantity across them; a pressure outlet with zero gauge pressure is imposed at the downstream exit boundary and all wheelset surfaces are defined as either stationary or moving (for rotating case) no-slip walls. The wheel rotation effect is implemented by imposing the corresponding rotation velocity on the solid surface. Numerical calculations are performed at two freestream velocities of 30 and $70 \mathrm{~m} / \mathrm{s}$. The resulting Reynolds numbers (based on the axle diameter and the freestream properties) are 36,000 and 83,900, which are within the subcritical Reynolds number regime. As well known, for a uniform stream passing over the axle of cylindrical shape, the boundary layer separation is laminar and vortex shedding occurs in the wake area with a Strouhal number of around 0.2 in the subcritical flow regime.

A rigorous grid convergence study for a complex geometry case is difficult to achieve because of the large calculations required for the unsteady flow. As a main part of the wheelset, the axle is a typical circular cylinder, from which numerical and experimental data are available for comparison. Therefore, a mesh refinement study has been performed on a circular cylinder at the same flow condition and is used to provide guidelines for the wheelset mesh generation. The influence of spatial resolutions has been compared by using different grid points in the $x-y$ plane (cases named 'Coarse', 'Baseline' and 'Fine') and along the spanwise ( $z$ ) direction. The time resolution was 
examined through reducing the timestep size by a factor of five. The effects of changes in $y^{+}$(the dimensionless first-cell spacing) were also analyzed. The Strouhal number (St) of the shedding frequency, root-mean-square values of the fluctuating lift coefficient $\left(C_{L}^{\prime}\right)$ and drag coefficient $\left(C_{D}^{\prime}\right)$, mean drag coefficient $\left(\bar{C}_{D}\right)$, the separation angle $(\theta)$ and the dimensionless recirculation length $\left(L_{r} / D\right)$ for each case have been computed and all the predicted results are found to exhibit a certain degree of grid convergence. The 'Baseline' case is demonstrated to have adequate resolution and is used to guide the generation of grids in the simulations here.

Based on the results of the grid convergence study for the cylinder case, a fully structured mesh is generated around the wheelset (displayed in Fig. 2) with resolutions similar to the cylinder 'Baseline' grids. The cell size on the axle surface is implemented as $0.4 \mathrm{~mm}$ around the perimeter and $0.8 \mathrm{~mm}$ in the spanwise direction. The maximum cell size on the wheel surface is $0.9 \mathrm{~mm}$. The mesh in the corner area between the wheel and axle is refined with double grid points in the wheel radial direction and the axle axial direction. For the case with inlet velocity of $30 \mathrm{~m} / \mathrm{s}$ as an example, the distance from the wheelset to the nearest grid point is set as $10^{-5} \mathrm{~m}$ and stretched with a growth ratio of 1.1 inside the boundary layer, yielding a maximum value of $y^{+}$less than 1 . For Reynolds-averaged Navier-Stokes (RANS) simulation or detached-eddy simulation (DES), the $y^{+}$in the first near-wall cell should be very small (on the order of $y^{+}=1$ ) to ensure the turbulence models employed inside the viscous sublayer to account for the low-Reynolds number effects. The total number of grid points in the entire domain is 5.5 million, distributed mainly in the near-wall and wake regions around the wheelset. The physical timestep size is $10^{-5} \mathrm{~s}$ which gives an adequate temporal resolution for the simulation with respect to the Courant-Friedrichs-Lewy values of less than 2 considering the implicit time marching scheme used here.

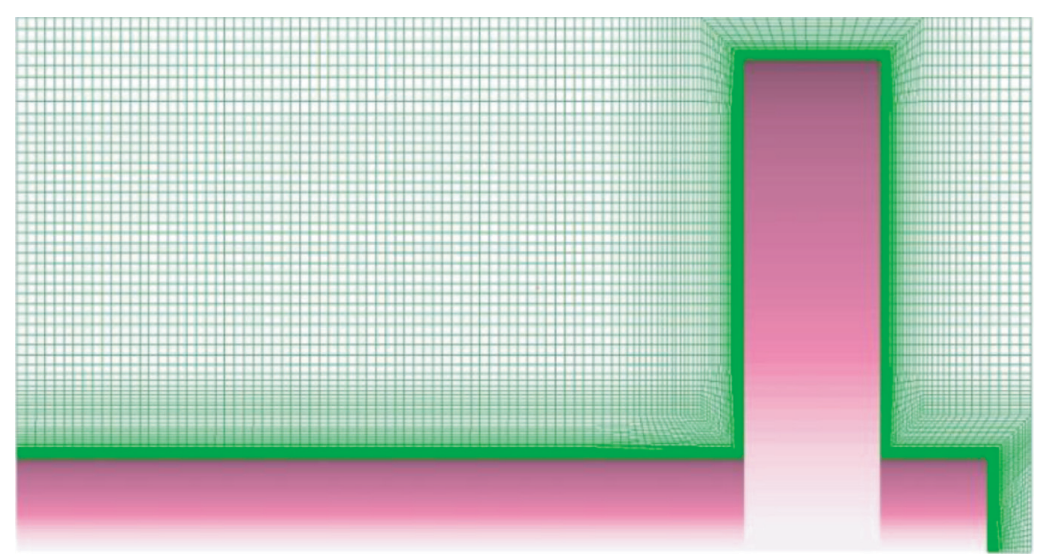

Figure 2: Mesh topology. 


\section{AERODYNAMIC RESULTS}

In order to understand the flow behaviour around the wheelset, the simulation results of the instantaneous iso-surfaces of $Q$-criterion and the vorticity fields are displayed. The gauge pressure at the wake positions as well as the lift and drag coefficients from the different geometries are compared. The numerical calculations presented in this section are based on the non-rotating (stationary) geometries with inflow velocity of $30 \mathrm{~m} / \mathrm{s}$.

\subsection{Properties of DDES model}

Detail descriptions on delayed detached-eddy simulation model were introduced in [7]. In simulations of turbulent flow using DES, the mesh should be so designed as to ensure that the boundary layer region is modelled by RANS while large-eddy simulation (LES) is only switched on outside the boundary layer. In DDES, the switch between RANS and LES is controlled by a redefined length scale $(\tilde{d})$ which depends not only on the cell wall distance and grid spacing but also on the time-dependent eddy-viscosity field. In order to check the RANS/LES switching of the DDES scheme, Fig. 3 illustrates the radial profiles of the mean velocity, the model length scale ratio $\left(r_{d}\right)$, DDES function $\left(1-f_{d}\right)$ and the ratio of the modified length scale to wall distance $(\tilde{d} / d)$ at $\theta=60^{\circ}$ (measured clockwise from the front stagnation point) at the mid-span locations of the wheel and the axle. The delay function $f_{d}$ is given by

$$
f_{d} \equiv 1-\tanh \left(\left(8 r_{d}\right)^{3}\right)
$$

in which the model length scale ratio $r_{d}$ applied to any eddy-viscosity model becomes slightly more robust in the irrotational regions and is represented as

$$
r_{d} \equiv \frac{v_{t}+v}{\sqrt{U_{i, j} U_{i, j}} k^{2} d^{2}},
$$

where $U_{i, j}=\partial U_{i} / \partial x_{j}$ is the velocity gradients and the molecular kinematic viscosity $v$ is employed to rectify the very-near-wall behaviour through keeping $r_{d}$ away from zero. The function $\left(1-f_{d}\right)$ approaches zero in the LES region. As shown in the figure, the wheel mid-span represents the $x-y$ surface from the midpoint along the axial direction, whereas the axle mid-span is defined at the half-length position of the axle segment inside the wheel. In terms of $r / D$ (the dimensionless distance to the wall surface) in the abscissa, the boundary layer (i.e. based on $U / U_{\infty}$ ) extends to 0.003 and RANS/LES switching occurs (the location where $\tilde{d} / d$ becomes less than 1) around 0.008 at the wheel mid-plane. At the axle mid-plane the boundary layer extends to 0.007 and RANS/LES switching occurs at 0.033. It is shown that the RANS-LES interface remains well outside the boundary layer and the DDES delay function $f_{d}$ reaches 1 within the LES region. Therefore, it is confirmed that the RANS method is imposed over the entire boundary layer and the LES treatment is applied elsewhere when using the DDES model in the simulation. 


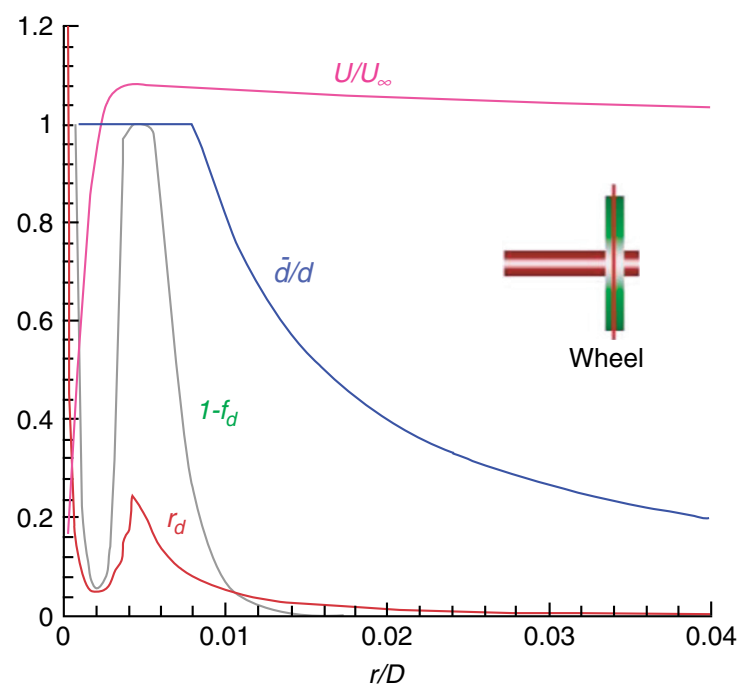

(a) Wheel mid-span

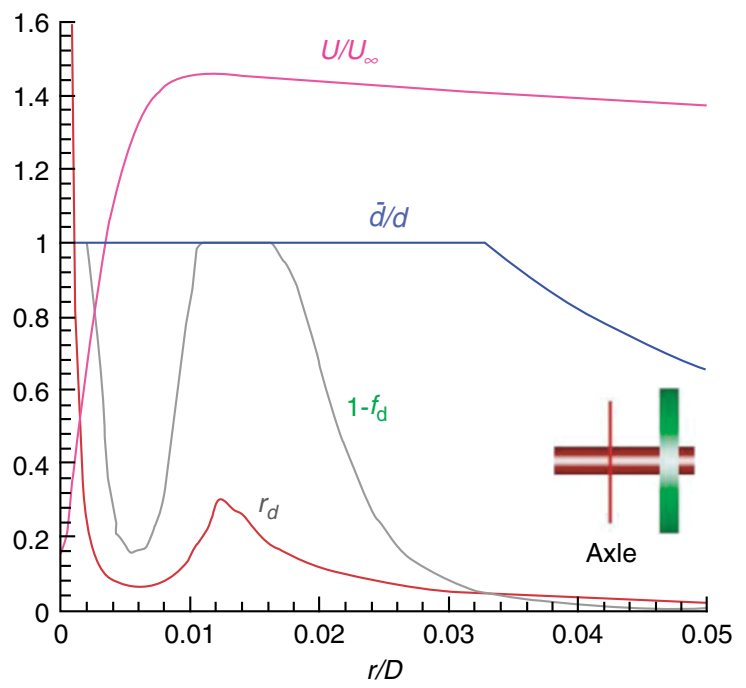

(b) Axle mid-span

Figure 3: $\quad$ DDES model properties $\left(\theta=60^{\circ}\right)$.

\subsection{Flow field}

Fig. 4 visualizes the wheelset's wake structures represented by the iso-surfaces of normalized $Q$-criterion value of 50 (based on $Q\left[\left(U_{0} / D\right)^{2}\right]$, where $U_{0}$ is the freestream velocity and $D$ the wheel diameter). They are coloured by the velocity magnitude 


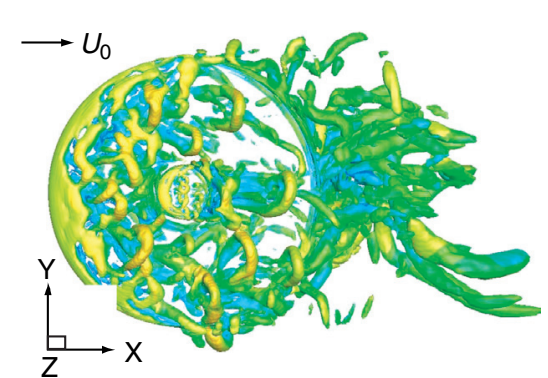

(a) Side view

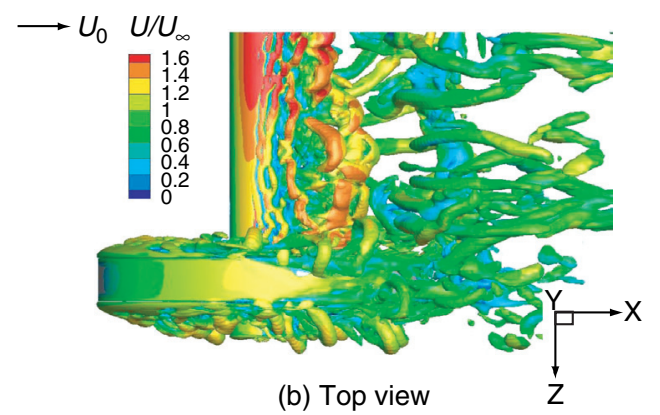

(b) Top view

Figure 4: Iso-surfaces of the instantaneous normalized $Q$-criterion ( around the tly velociity.

normalized with freestream velocity. It can be seen that flow separates at the wheel front edges, and reattaches within a very short distance on the wheel flat side surface, forming a crescent-shaped separation bubble; the subsequent horseshoe-shaped eddies are formed and carried downstream. In the axle wake, two-dimensional spanwise vortices are generated first straight behind the axle, followed by streamwise vortices developed further downstream, suggesting a three-dimensional character of the solutions in the wheelset wake area. Additionally, compared to the flow developed around the wheel, a higher level of velocity magnitude occurs in the axle wake, indicating a significantly energetic flow generated there.

The instantaneous non-dimensional spanwise vorticity fields $\left(\omega_{z}=\right.$ $\left.(\partial V / \partial x-\partial U / \partial y) D / U_{\infty}\right)$ in the wake area behind the axle and wheel are displayed in Fig. 5. This reveals that the flow behaviour around the axle is different from that around the wheel: the organized vortex structures in the axle wake are clearly formed and dominated by a large alternating shedding while the wheel wake is highly turbulent with

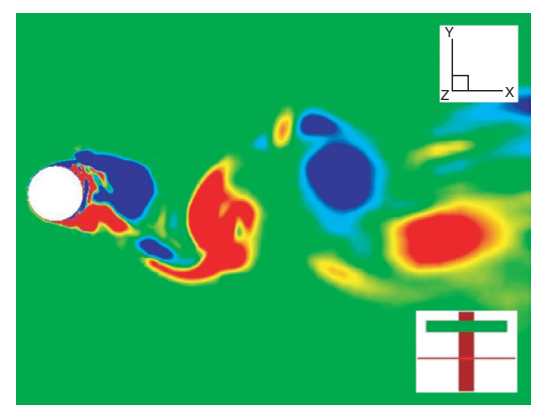

(a) Axle mid-span

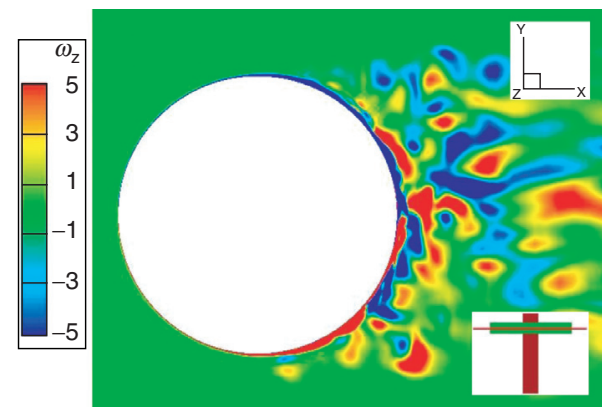

(b) Wheel mid-span

Figure 5: Contours of instantaneous spanwise vorticity fields in vertical planes through centre of axle and wheel (side views). 
eddies of different scales. This is understandable as the axle provides the appropriate spanwise correlation length to generate vortex shedding whereas the wheel is threedimensional with small aspect ratio, the flow separation from the side surface influences the vortices shed behind the wheel, causing the flow structure behind the wheel to be fully three-dimensional and less organized, with various scales.

Fig. 6 displays the instantaneous non-dimensional spanwise vorticity fields $\left(\omega_{z}\right)$ in the axle wake at different distances to the wheel inner surface. It can be seen from Fig. 6(a) that in the axle wake with the gap of $5 \mathrm{~mm}$ away from the wheel inner surface, the vortices shed from the axle interact with the vortices generated from the wheel circumferential edge; they deform largely and are merged into the eddies formed behind the wheelset, leading to the synchronized behaviour of the wheelset wake. Close to the wheel (shown in Fig. 6b), the interaction of the vortices generated from the axle and wheel becomes weaker and the wake is dominated by flow separations occurring on the wheel top and bottom rim edges.

Fig. 7 shows the power spectral density (PSD) of the gauge pressure at different positions in the wheelset wake. A tonal peak at $311 \mathrm{~Hz}$ is clearly seen in Fig. 7(a) showing the PSD at a point with a distance of one axle radius from the top side of the axle at mid-span. This frequency is associated with the vortex shedding from the axle, and the corresponding Strouhal number (non-dimensionalized by the freestream velocity and the axle diameter) is 0.182 . Fig. 7(b) illustrates that the PSD of the pressure at a point with a distance of one wheel radius away from the top of the wheel at midspan is broadband and its amplitude is much lower than that in the axle wake. This indicates that flow behind the wheel is fully three-dimensional and the wake contains the turbulent structures with various scales. Fig. 7(c) shows the results for points located at $3 \mathrm{~mm}$ and $13 \mathrm{~mm}$ away from the wheel inner rim in line with the top of the axle. Two peaks appear at the frequency of $311 \mathrm{~Hz}$ and $622 \mathrm{~Hz}$, and these correspond respectively to the axle vortex shedding and the interaction between the vortex shedding from the axle and the unsteady flow around the wheel in the drag direction. It is noted that closer

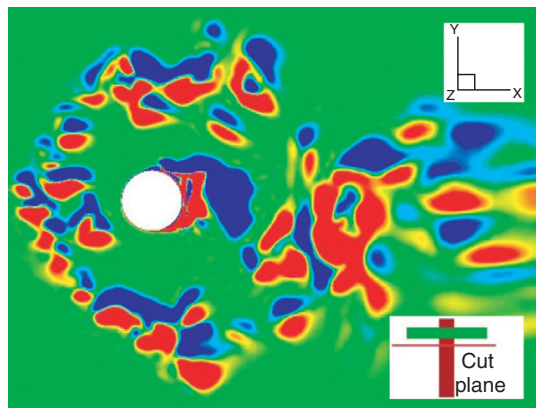

(a) $5 \mathrm{~mm}$ from wheel

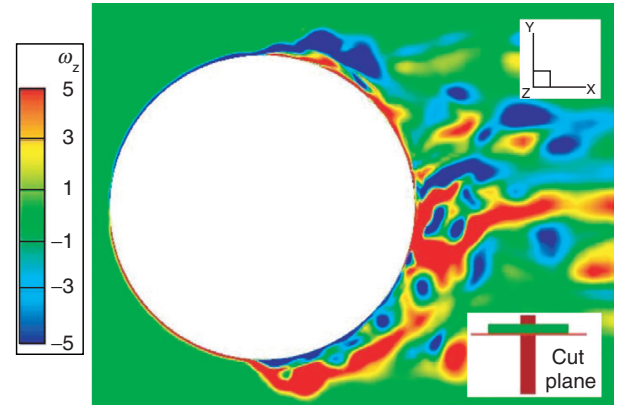

(b) Close to wheel

Figure 6: Contours of instantaneous spanwise vorticity fields in vertical planes through corner of wheel and axle (side views). 


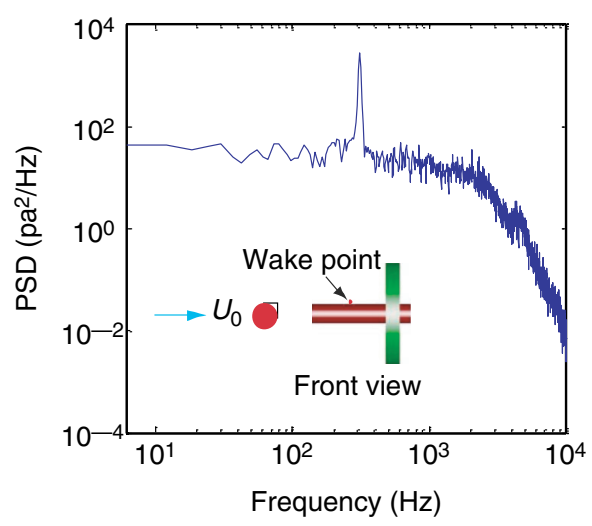

(a) Axle mid-span

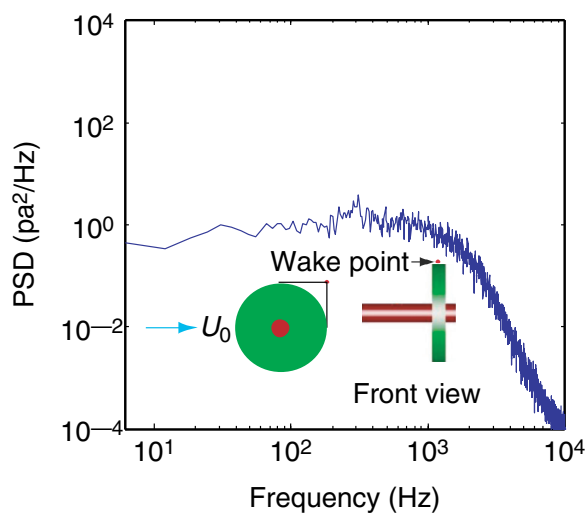

(b) Wheel mid-span

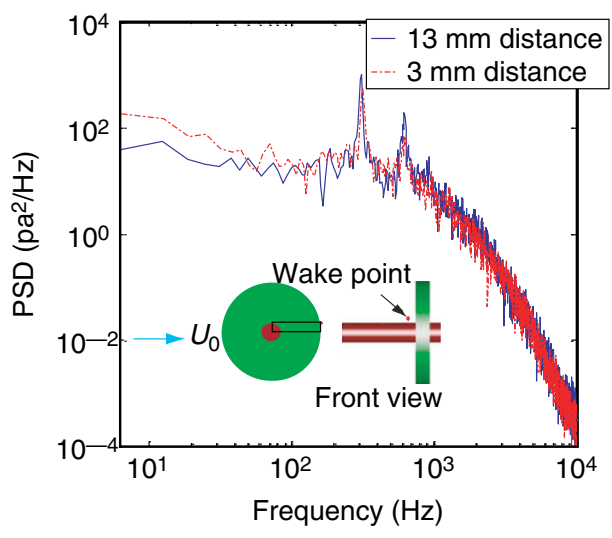

(c) Axle near wheel

Figure 7: Power spectral density of pressure at wheelset wake positions.

to the wheel, the tonal peak amplitude of the pressure signal becomes lower; particularly, the second peak at $622 \mathrm{~Hz}$ drops by more than the first one at $311 \mathrm{~Hz}$. This is because the vortex shedding from the axle is less strong there and much weaker impact is generated by the axle shedding vortices on the unsteady flow around wheel, resulting in the less drag fluctuation produced in the wheel-axle corner region.

\subsection{Lift and drag coefficient}

Fig. 8 depicts the PSDs of the lift and drag coefficients of the wheelset. In order to provide insight, results are shown for the separate components for the wheel and axle when they form part of the wheelset. A clear peak appears in all lift coefficient PSDs at $311 \mathrm{~Hz}$, corresponding to a Strouhal number (non-dimensionalized by the freestream velocity and the axle diameter) of 0.182 . This peak is associated with the axle primary 


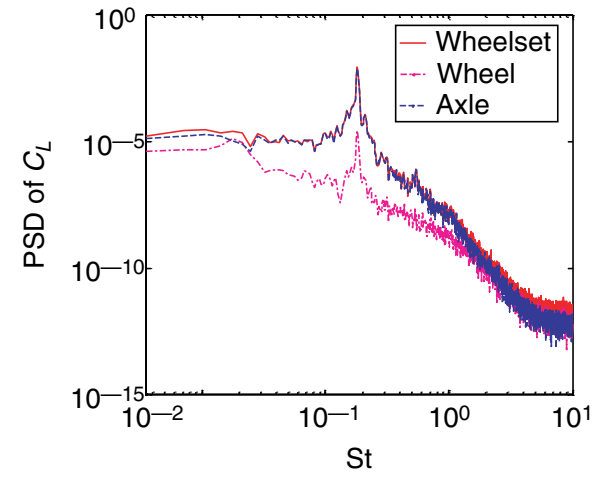

(a) Lift coefficient

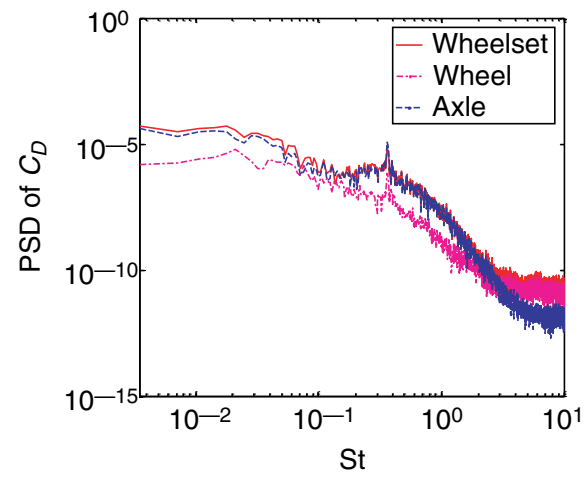

(b) Drag coefficient

Figure 8: $\quad$ Power spectral density of lift and drag coefficient.

shedding frequency. By comparison, another lower peak is observed in the drag coefficient curves at twice the shedding frequency. As known, the aerodynamic lift force acting in the transverse direction normal to the flow fluctuates with a larger amplitude at half the frequency of the drag force which acts in the streamwise direction parallel to the flow. It can be seen that the flow around the wheelset and that around its components have the same shedding frequencies. This is because the periodic, alternating vortex shedding produced from the axle dominates the wake unsteadiness and interacts with the wall boundary layer and unsteady flow separated from the wheel (consistent with Figs. 6 and 7c), thereby the resulting wake induces the regular fluctuating forces on the whole wheelset. Compared to the axle where the massive vortex shedding occurs, the wheel has the same dominant frequencies in the lift and drag forces, but with much lower amplitude. Considering the axle and wheel in isolation from each other, it should be noted that the main peak of the drag force $(S t=0.36)$ cannot be identified for the isolated axle case while the flow around the isolated wheel is fully irregular with no periodic vortex shedding, as shown in Fig. 9.

\section{AEROACOUSTIC RESULTS}

Based on the near-field flow data obtained from the CFD calculations, the FW-H method can predict far-field noise signals by equivalent acoustic sources as stated previously. There are 22,016 panels around the wheel and 15,232 panels on the axle which account for the acoustic sources on the solid integration surfaces. The receivers are distributed uniformly along a circumference with $2.5 \mathrm{~m}$ radius at an interval of $5^{\circ}$ as sketched in Fig. 10 to measure the noise directivity from the wheelset centreline.

\subsection{Acoustic spectra computation}

The CFD simulations were run for $0.93 \mathrm{~s}$ corresponding to 20 times the flow-through time. The length of the time signal used as input to the FW-H method for noise calculation is related to the last $0.55 \mathrm{~s}$ of the computation. The PSD is computed from 


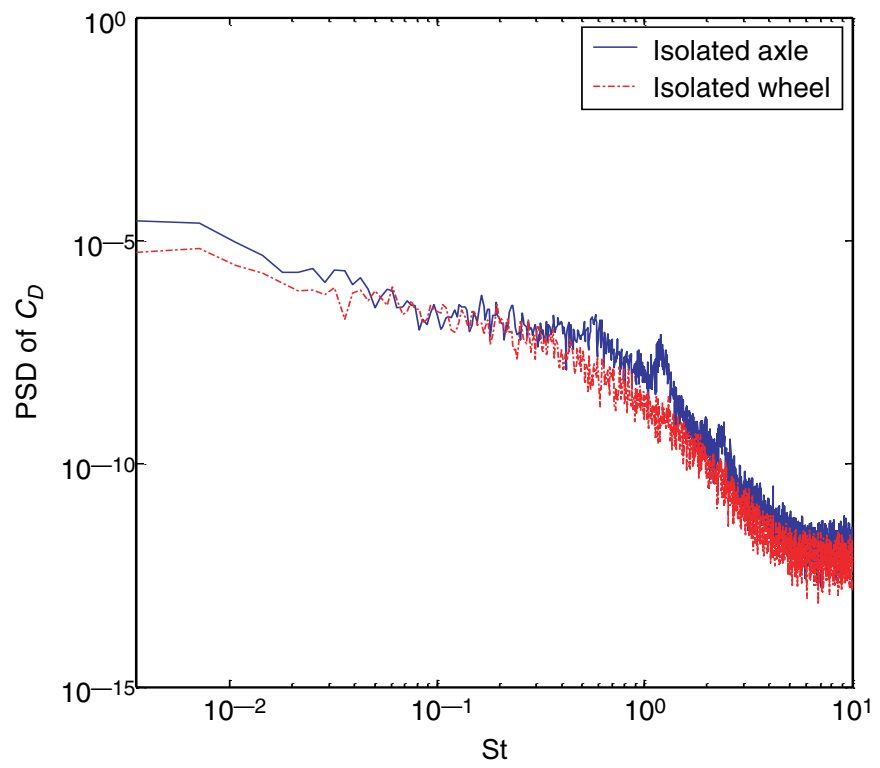

Figure 9: Power spectral density of drag coefficient.
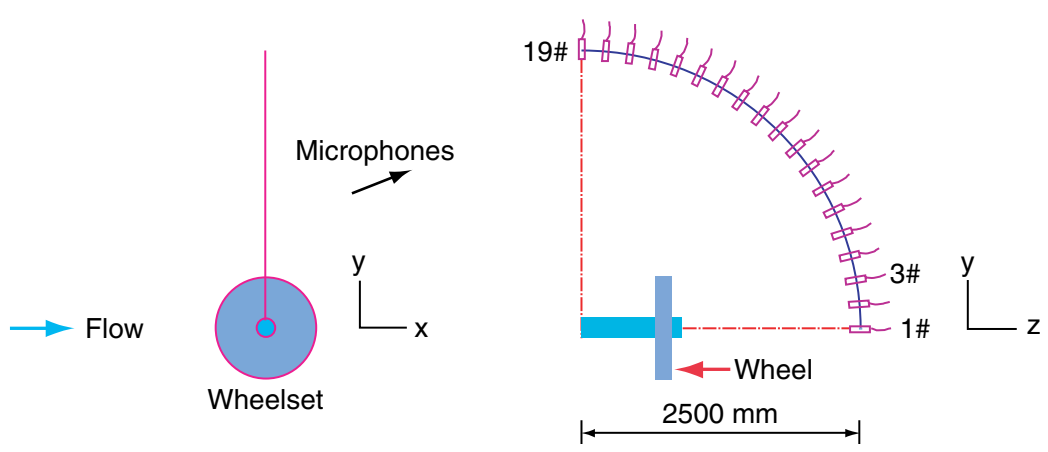

Figure 10: Sketch of receiver locations.

the predicted far-field noise time history by the Welch's method and averaged over $50 \%$ overlapping segments using a Hanning window applied to 5 segments, giving a frequency resolution of $6 \mathrm{~Hz}$.

Based on the numerical simulations on the symmetrical half wheelset and whole isolated wheel (i.e. without the axle) cases, Fig. 11 shows the spectra of the noise radiated from the non-rotating wheelset and an isolated wheel at three receivers as 


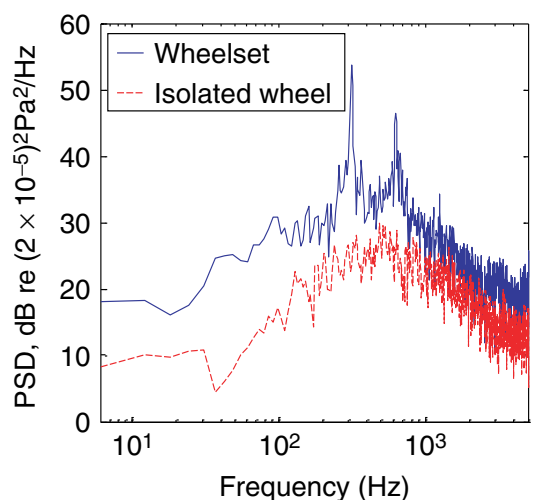

(a) Receiver 3

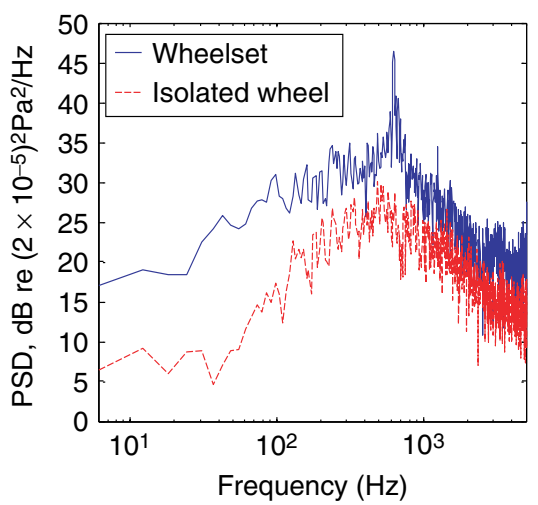

(c) Receiver 1

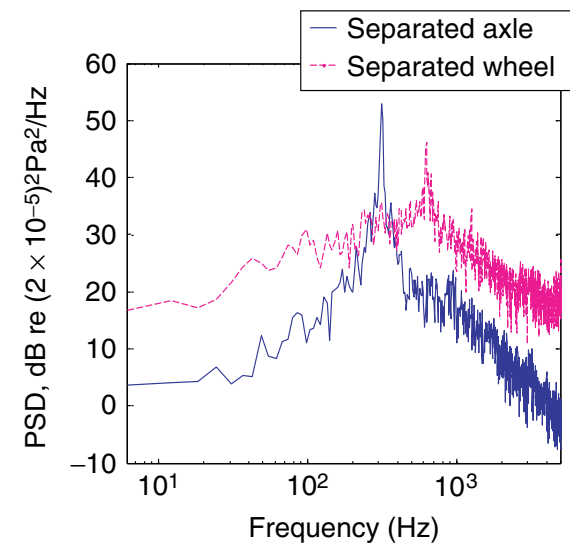

(b) Wheelset components (receiver 3)

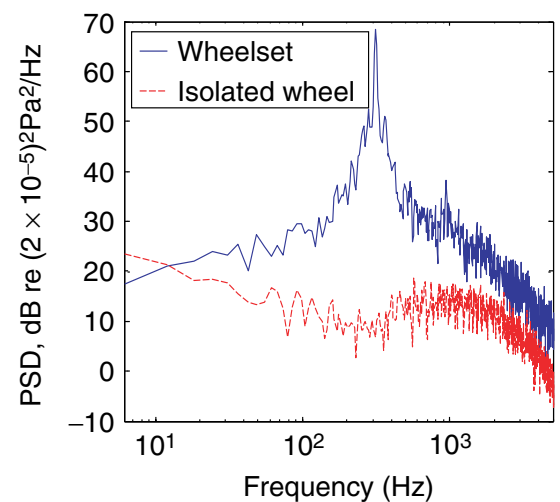

(d) Receiver 19

Figure 11: Spectra of acoustic pressure at far-field receivers $\left(U_{\infty}=30 \mathrm{~m} / \mathrm{s}\right)$.

well as the noise radiated by components of the wheelset (separate components of the wheel and axle) at one receiver in the $z-y$ plane as described in Fig. 10. The largest drag dipole component is found at receiver 1 while receiver 19 shows the highest lift dipole component. At receiver 3 both the lift and drag dipole components are found, as will be discussed subsequently. From the wheelset case in Fig. 11(a), it is noted that the tonal peaks appear at the frequencies of $311 \mathrm{~Hz}$ and $622 \mathrm{~Hz}$ at receiver 3 which is located $0.434 \mathrm{~m}$ above the axle axis. The primary peak corresponds to the oscillating lift forces exerted back on the fluid around the wheelset and the peak at the second harmonic is associated with the oscillating drag forces. As mentioned earlier, the frequency of the fluctuating drag is twice that of the fluctuating lift. 
Fig. 11(b) compares the spectra of the noise radiated from the sources on the axle and the wheel separately (as part of the wheelset) at receiver 3. Again, two tonal noises are found with dominant frequencies corresponding to the lift and drag forces respectively. Therefore, in the vertical $z-y$ plane normal to the flow direction the sound radiation produced from the separated wheel in the presence of the axle is mainly associated with the drag forces, whereas the noise generated by the separated axle in the presence of the wheel mainly corresponds to lift forces. As a result, the level of the second harmonic is largest at receiver 1 (shown in Fig. 11c) while that of the primary shedding frequency is highest at receiver 19 (shown in Fig. 11d). Compared with the wheelset case, the noise radiated from the isolated wheel is more broadband with a lower spectral level, resulting from the irregular flow with no periodic shedding generated around it as stated earlier.

Furthermore, to improve the completeness of current analyses, the spectra of the noise generated from a rotating wheelset $\left(U_{\infty}=30 \mathrm{~m} / \mathrm{s}\right)$ and a non-rotating wheelset $\left(U_{\infty}=70 \mathrm{~m} / \mathrm{s}\right.$, closer to the high-speed train case) at receiver 3 are compared with the non-rotating wheelset case in Fig. 12. It is found that the frequencies of the two dominant peaks in the rotating wheelset case are both around $6 \mathrm{~Hz}$ higher but with similar amplitude compared to those from the non-rotating wheelset as illustrated in Fig. 12(a). Thus, the rotation of the wheelset is expected to have negligible influence on the noise generation, as confirmed below. Moreover, Fig. 12(b) shows that as the inflow velocity increases to $70 \mathrm{~m} / \mathrm{s}$, the noise levels increase greatly and the frequencies of the two spectral peaks $(726 \mathrm{~Hz}$ and $1447 \mathrm{~Hz}$ ) increase in proportion to the flow speed, corresponding to an invariant Strouhal number characterized by regular vortex shedding.

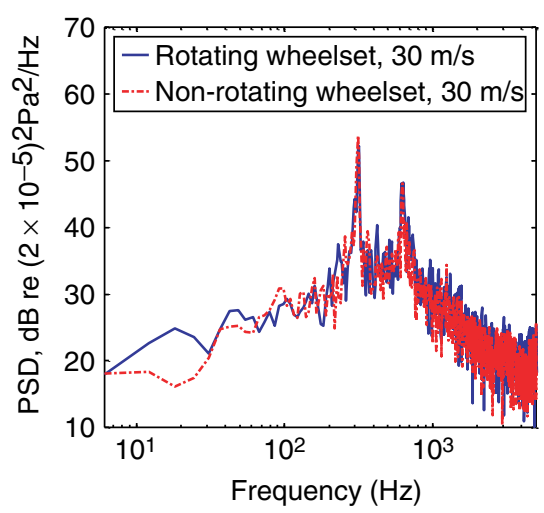

(a) Rotating and non-rotating wheelset $(30 \mathrm{~m} / \mathrm{s})$

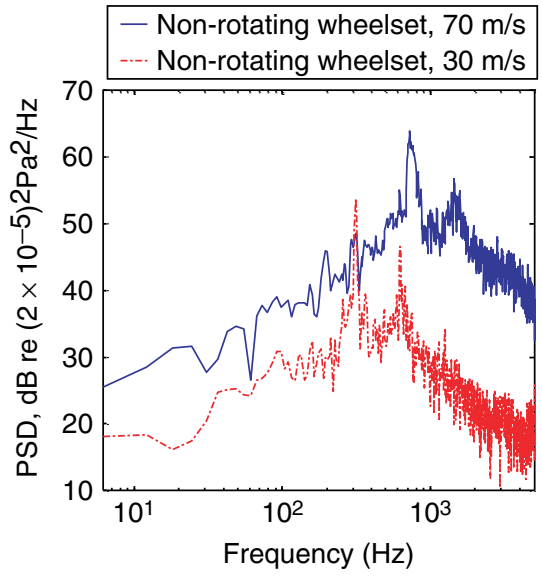

(b) Non-rotating wheelset (30 and $70 \mathrm{~m} / \mathrm{s})$

Figure 12: Spectra of acoustic pressure at receiver 3 . 


\subsection{Experimental verification}

An experimental measurement of sound generated by flow past a non-rotating wheelset was made in an open-jet anechoic wind tunnel. Fig. 13 displays the experiment setup of which the test model is mounted in the working section on a rigid baffle. A 1:10 scale half-wheelset is immersed within the core flow and the remaining parts are wrapped with sound-absorbing sponge to suppress the aerodynamic noise generated by vortex shedding from such a portion. The exit nozzle has a rectangular cross-section $(350 \mathrm{~mm} \times$ $500 \mathrm{~mm}$, width by height) and the flow speed is $30 \mathrm{~m} / \mathrm{s}$ with the turbulence level in the jet core below $0.3 \%$. In accordance with the numerical predictions, two receivers identified as 'top microphone' and 'side microphone' are located at $(-18,1375,31.3)$ and $(0,185,2211.3)$, of which the dimensions in millimetres and the coordinates (see Fig. 13) with the origin at the centre of the axle outer end surface. Corresponding to the frequency resolution used in the simulation, the PSD of test data is also computed by the Welch's method with $6 \mathrm{~Hz}$ bandwidth.

Fig. 14 displays the spectra of the radiated noise at the two receivers. Due to a high background noise in the low frequency range generated from the nozzle itself, results are only considered above $100 \mathrm{~Hz}$. Fig. 14(a) compares the predicted and measured PSD levels for the top microphone. Good agreement can be found for the dominant frequency of tonal noise and the shape of the spectrum; even a small peak around $960 \mathrm{~Hz}$ (third harmonic) is accurately predicted in the computation. In the experiment a low amplitude peak appears around $234 \mathrm{~Hz}$, which is not observed in the simulation. This peak may be associated with the noise contribution from the interaction between the axle wake and the baffle plate, i.e. the vortex shedding in the axle wake region close to the baffle is decreased by the unsteady flow developed on the rigid plate and thereby the corresponding shedding frequency is reduced. This same phenomenon has been found in the experiments of flow-induced noise from wall-mounted cylinders [11]. Compared with the experimental data, the tonal peak is higher in the calculated spectrum. Again, this is likely to be due to the solid wall
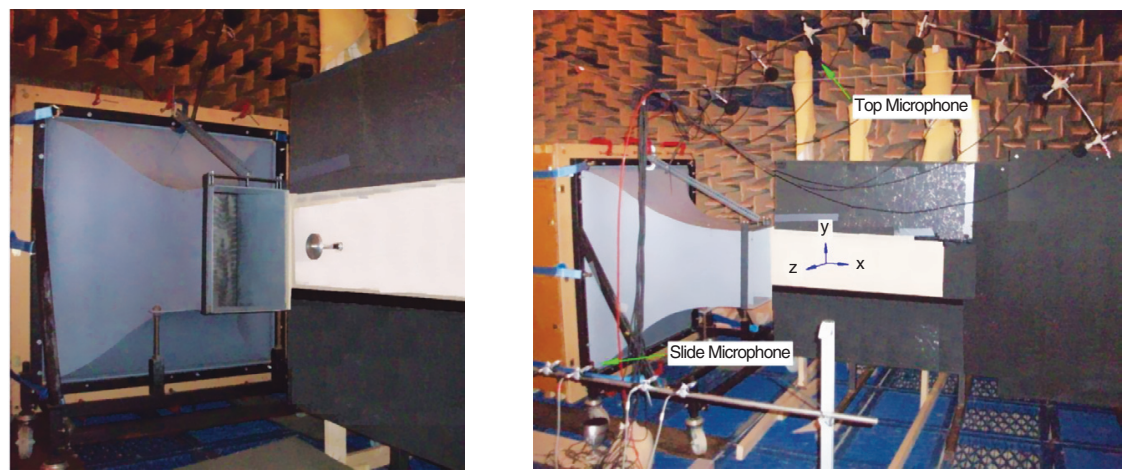

Figure 13: Experiment setup in the anechoic chamber. 


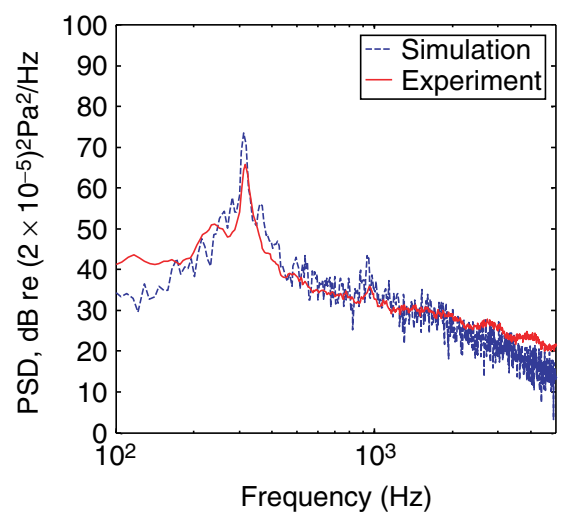

(a) Top receiver

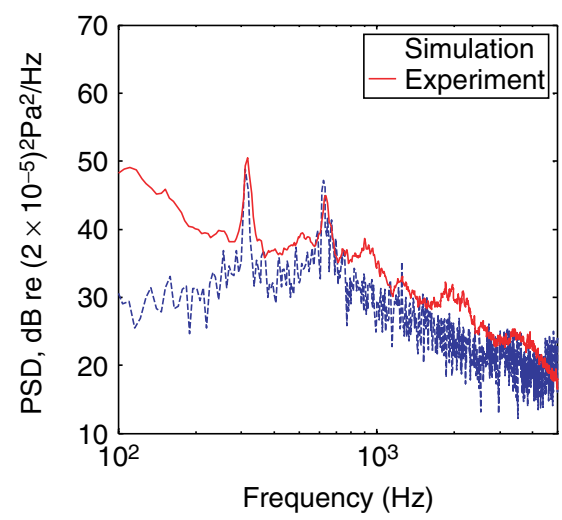

(b) Side receiver

Figure 14: Comparisons of spectra of far-field sound from simulations and experiments.

(rigid baffle) used in the experiment which weakens the coherent vortex shedding from the axle compared to the symmetric boundary conditions with stronger spanwise uniformity applied in the simulation.

Fig. 14(b) shows the spectra of the radiated noise from prediction and experiment at the side microphone. Note that the experimental noise levels are systematically higher than the predictions at the side microphone due to the additional noise contributions caused by reflections from the baffle plate. The two tonal peaks in the measurements correspond fairly well with the noise predictions: both the dominant frequency values and the harmonic behaviour. This indicates that the sound reflection from the baffle plate has a little effect on the frequencies of the main peak and harmonics caused by the vortex shedding from the wheelset.

\subsection{Acoustic directivity}

The directivity of radiated noise in the far-field is obtained based on the overall calculated sound pressure level which is determined from the PSDs over the frequency range below $5 \mathrm{kHz}$. As the symmetry plane is used, only the flow data of half geometry are available and applied for the noise prediction. Thus, the sound pressure level from the whole geometry are given by $L_{p}=10 \log \left(10^{L_{p 1 / 10}}+10^{L_{p 2} / 10}\right)$, where $L_{p_{1}}$ and $L_{p_{2}}$ are the sound pressure levels of two receivers located symmetrically along the symmetry plane with the same sound source from the half geometry. Fig. 15 shows the noise directivities from the rotating and non-rotating wheelsets. As the inflow speed increases from $30 \mathrm{~m} / \mathrm{s}$ to $70 \mathrm{~m} / \mathrm{s}$, the noise levels are increased by $18.5 \mathrm{~dB}$ with the similar directivity pattern. This corresponds to an increase in the radiated sound power in proportion to about the sixth power of the flow speed as expected for an aeroacoustic dipole source. 


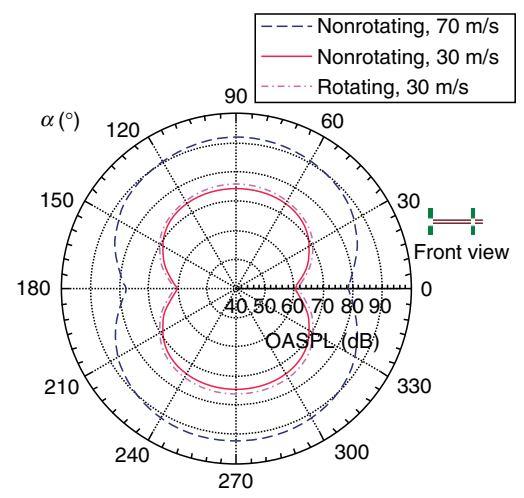

(a) Vertical $z-y$ plane

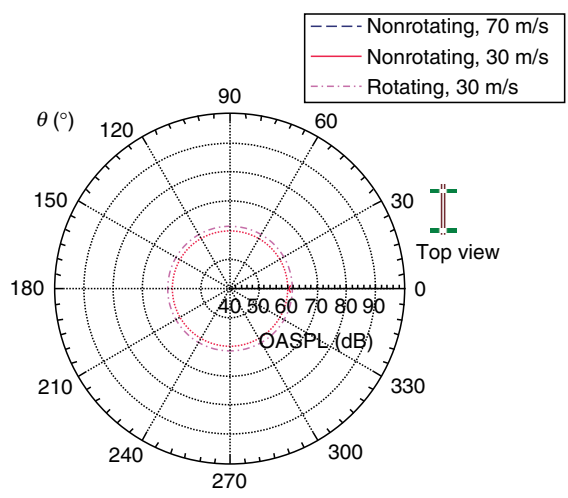

(b) Horizontal $x-z$ plane

Figure 15: Noise directivity from rotating and nonrotating wheelset.

Based on the noise directivities shown in Fig. 15 and Fig. 16(a), it can be concluded that the dipole patterns of the noise generated from the rotating and non-rotating wheelsets are very close, with noise levels that are about $2 \mathrm{~dB}$ higher for the rotating case. Fig. 16(b) shows the noise directivity from the separate components from the wheel and axle of the rotating and non-rotating wheelsets. It can be seen that the vertical dipole pattern of the non-rotating wheel is inclined upwards toward the inflow direction for the rotating wheel and the difference in the noise levels between them is small. Compared to the separated wheel, the noise generation from the axle part is much larger. This leads to the vertical dipole pattern of noise radiation from the rotating wheelset presented in Fig. 17, which clearly shows that the lift dipole dominates the

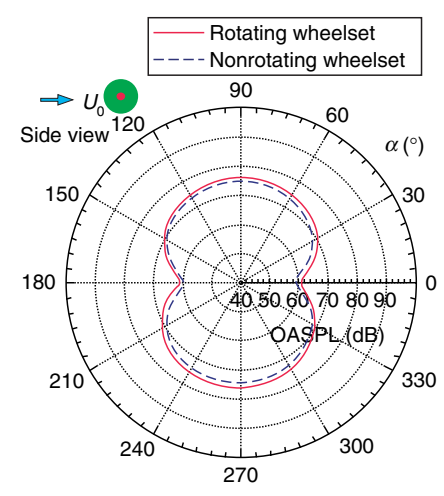

(a) Whole wheelset

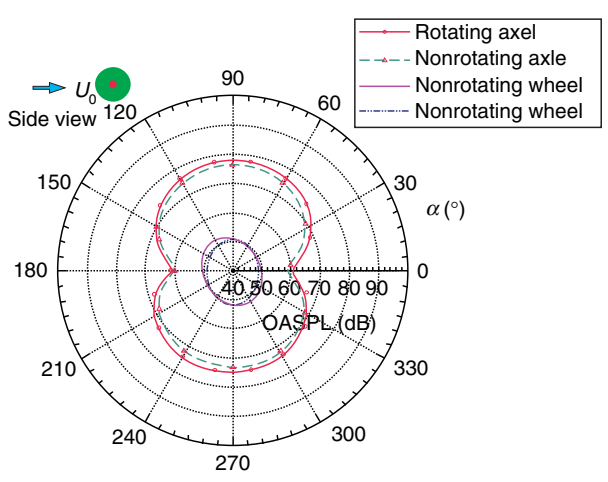

(b) Separated wheel and axle

Figure 16: Noise directivity for rotating and non-rotating wheelset (streamwise $x-y$ plane). 

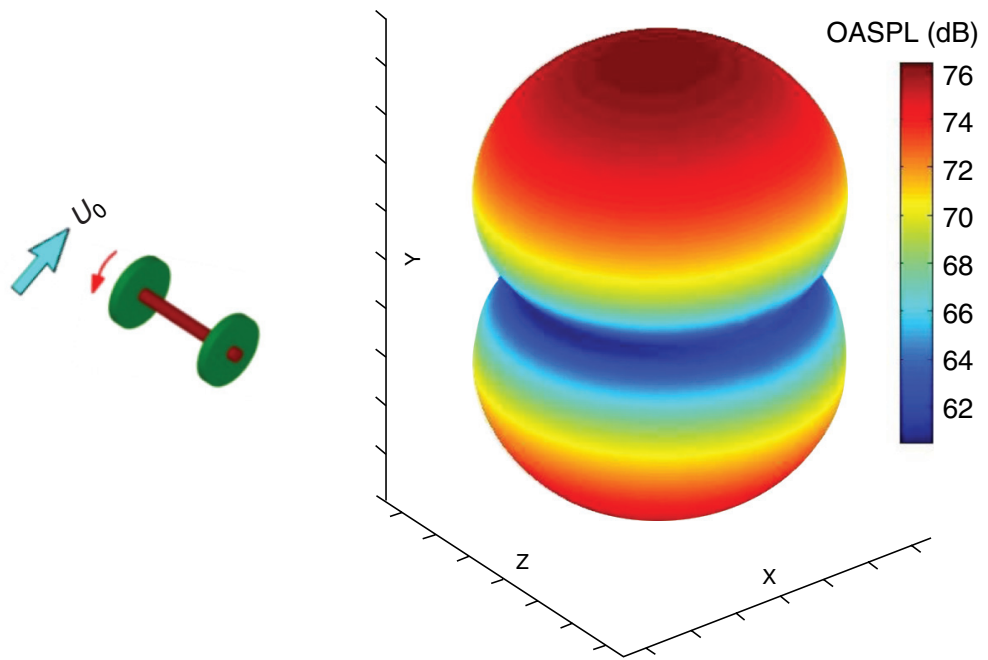

Figure 17: Three-dimensional noise directivity for a rotating wheelset.

directivity shape (centred at $40 \mathrm{~dB}$ ). The non-rotating wheelset case has a similar vertical dipole directivity pattern which can be indicated from Fig. 15. Additionally, compared with the non-rotating case, the noise generation from the rotating axle is slightly larger as a consequence of the stronger flow-axle interaction caused by the rotation effect, making the noise of the rotating wheelset generally higher as mentioned earlier.

\section{CONCLUSIONS}

The aerodynamic and aeroacoustic behaviour of the flow past an isolated wheelset have been studied using the DDES model and FW-H acoustic analogy. It is found that the turbulent flow around the wheelset has a complicated three-dimensional wake structure: both streamwise and spanwise vortices are formed due to the vortex shedding and flow separation around it. In aeroacoustic prediction, the noise calculation compares fairly well with the experimental measurements. For both the rotating and the non-rotating wheelsets, tonal noise is generated with dominant frequencies corresponding to the oscillating lift and drag forces; a vertical dipole pattern of noise directivity is predicted with slightly higher level for the rotating case whereas the rotation effect has little effect on the noise generation from an isolated wheelset, at least in the present case where the ground effect is neglected. As the inflow velocity increases, the frequencies of spectral peaks increase corresponding to an invariant Strouhal number characterized by regular vortex shedding and the noise levels increase considerably.

In order to interpret these results for a full-scale case, it may be noted that the Reynolds number in reality is much higher; thus, the tonal components are likely to be less significant and the broadband component is likely to increase in importance; the 
more complex geometry will also lead to more complex flow structures and this will affect the noise radiated. Such factors will need to be accounted for in future work.

\section{ACKNOWLEDGEMENTS}

The numerical simulations were performed on the Iridis4 cluster managed by University of Southampton. The first author would like to thank Koen van Mierlo for help in post-processing of simulated data and Eduardo Latorre Iglesias for the assistance in the experimental measurements. The research studentship provided by University of Southampton is gratefully appreciated.

\section{REFERENCES}

[1] D.J. Thompson. Railway noise and vibration: mechanisms, modelling and means of control. Elsevier, Oxford, UK, 2008.

[2] C. Mellet, F. Letourneaux, F. Poisson, C. Talotte. High speed train noise emission: Latest investigation of the aerodynamic/rolling noise contribution. Journal of Sound and Vibration, 2006, 293: 535-546.

[3] C. Talotte, P.E. Gautier, D.J. Thompson, C. Hanson. Identification, modelling and reduction potential of railway noise sources: a critical survey. Journal of Sound and Vibration, 2003, 267: 447-468.

[4] M. Meskine, F. Pérot, M.S. Kim, D. M. Freed, S. Senthooran, Z. Sugiyama, F. Polidoro, S. Gautier. Community noise prediction of digital high speed train using LBM. AIAA Paper 2013-2015, 19 ${ }^{\text {th }}$ AIAA/CEAS Aeroacoustics Conference, Berlin, Germany, 2013.

[5] D.J. Thompson, M.G. Smith, F. Coudret. Application of a component-based approach to modelling the aerodynamic noise from high-speed trains. Proceedings of the $10^{\text {th }}$ International Workshop on Railway Noise, Nagahama, Japan, 2010: 449-456.

[6] P.R. Spalart, M.L. Shur, M.Kh. Strelets, A.K. Travin. Initial noise predictions for rudimentary landing gear. Journal of Sound and Vibration, 2011, 330, 4180-4195.

[7] P.R. Spalart, S. Deck, M.L. Shur, K.D. Squires, M.Kh. Strelets, A.K. Travin. A new version of detached-eddy simulation, resistant to ambiguous grid densities. Theoretical and Computational Fluid Dynamics, 2006, 20: 181-195.

[8] J.E. Ffowcs-Williams, D.L. Hawkings. Sound radiation from turbulence and surfaces in arbitrary motion. Philosophical Transactions of the Royal Society of London, 1969, 342: 264-321.

[9] K.S. Brentner, F. Farassat. Modelling aerodynamically generated sound of helicopter rotors. Progress in Aerospace Sciences, 2003, 39: 83-120.

[10] F. Farassat. Derivation of Formulations 1 and 1A of Farassat. NASA/TM-214853, 2007.

[11] D.J. Moreau, C.J. Doolan. The flow-induced sound of a wall-mounted finite length cylinder with circular and square cross-section. AIAA Paper 2013-2205, $19^{\text {th }}$ AIAA/CEAS Aeroacoustics Conference, Berlin, Germany, 2013. 
